\title{
Characterization of MED12, HMGA2, and FH (1) CrossMark alterations reveals molecular variability in uterine smooth muscle tumors
}

\author{
Netta Mäkinen ${ }^{1 *}$, Kati Kämpjärvi ${ }^{1}$, Norma Frizzell ${ }^{2}$, Ralf Bützow ${ }^{3}$ and Pia Vahteristo ${ }^{1 *}$
}

\begin{abstract}
Uterine smooth muscle tumors range from benign leiomyomas to malignant leiomyosarcomas. Based on numerous molecular studies, leiomyomas and leiomyosarcomas mostly lack shared mutations and the majority of tumors are believed to develop through distinct mechanisms. To further characterize the molecular variability among uterine smooth muscle tumors, and simultaneously insinuate their potential malignant progression, we examined the frequency of known genetic leiomyoma driver alterations (MED12 mutations, HMGA2 overexpression, biallelic FH inactivation) in 65 conventional leiomyomas, 94 histopathological leiomyoma variants (18 leiomyomas with bizarre nuclei, 22 cellular, 29 highly cellular, and 25 mitotically active leiomyomas), and 51 leiomyosarcomas. Of the 210 tumors analyzed, 107 had mutations in one of the three driver genes. No tumor had more than one mutation confirming that all alterations are mutually exclusive. MED12 mutations were the most common alterations in conventional and mitotically active leiomyomas and leiomyosarcomas, while leiomyomas with bizarre nuclei were most often FH deficient and cellular tumors showed frequent HMGA2 overexpression. Highly cellular leiomyomas displayed the least amount of alterations leaving the majority of tumors with no known driver aberration. Our results indicate that based on the molecular background, histopathological leiomyoma subtypes do not only differ from conventional leiomyomas, but also from each other. The presence of leiomyoma driver alterations in nearly one third of leiomyosarcomas suggests that some tumors arise through leiomyoma precursor lesion or that these mutations provide growth advantage also to highly aggressive cancers. It is clinically relevant to understand the molecular background of various smooth muscle tumor subtypes, as it may lead to improved diagnosis and personalized treatments in the future.
\end{abstract}

Keywords: Uterine leiomyoma, Histopathological uterine leiomyoma variants, Uterine leiomyosarcoma, MED12, $H M G A 2, F H$

\section{Background}

Uterine leiomyomas (ULs) are extremely common human neoplasms that originate from the smooth muscle cells of the myometrium. Due to their dependency on the ovarian steroids estrogen and progesterone, ULs occur in women of reproductive age and typically regress with the onset of menopause [1]. The life time prevalence of ULs is approximately $70 \%$ among white and more than $80 \%$ among black women [2]. Despite their benign nature, ULs

\footnotetext{
* Correspondence: netta.makinen@helsinki.fi; pia.vahteristo@helsinki.fi ${ }^{1}$ Research Programs Unit, Genome-Scale Biology Research Program and Medicum, Department of Medical and Clinical Genetics, FIN-00014 University of Helsinki, P.O. Box 63, Helsinki, Finland

Full list of author information is available at the end of the article
}

can cause considerable morbidity, such as heavy and prolonged menstrual flow, abdominal pain and discomfort, and reproductive dysfunction. They are also the single most prevalent cause for hysterectomy and pose a considerable socio-economic impact [3]. Based on histopathology, ULs can be divided into conventional leiomyomas and various relatively rare subtypes, such as leiomyomas with bizarre nuclei, cellular, and mitotically active leiomyomas [4]. Histopathological UL subtypes explain approximately $10 \%$ of all ULs. Although these subtypes mimic malignancy in one or more aspects, their behavior is thought to be benign.

Approximately $40 \%$ of leiomyomas harbor non-random cytogenetic rearrangements, of which the most common 
is a translocation between chromosome bands $12 \mathrm{q} 15$ and 14q24 leading to overexpression of high mobility group AT-hook 2 (HMGA2) [5-7]. Other chromosomal alterations include, for example, interstitial deletions in $7 \mathrm{q}$, rearrangements of $6 \mathrm{p} 21$, and trisomy 12 . Several independent studies representing various ethnic groups have shown that approximately $70 \%$ of ULs harbor specific mutations in mediator complex subunit 12 (MED12) [8-11]. All observed changes have been missense or small inframe insertion-deletion mutations in exons 1 and 2, affecting a highly conserved region of the gene. Subsequent studies have indicated that histopathological UL subtypes harbor significantly fewer MED12 mutations than conventional leiomyomas [12, 13]. Rarely, ULs can be associated with a familial Hereditary Leiomyomatosis and Renal Cell Cancer (HLRCC) syndrome. The syndrome is caused by heterozygous germline mutations in fumarate hydratase $(F H)$, which encodes an enzyme fumarase of tricarboxylic acid cycle [14]. Our recent highthroughput sequencing efforts have pointed to at least three distinct molecular subclasses among conventional ULs, each candidate subclass displaying a characteristic genetic driver aberration as well as unique global gene expression profile: MED12 mutation-positive, HMGA2overexpressing, and FH-deficient ULs [15, 16].

Uterine leiomyosarcomas (ULMSs) are rare, malignant smooth muscle tumors with a poor 5-year survival and high recurrence rate [17-20]. The majority of ULMSs occur in women $>50$ years of age with an annual incidence of $0.3-0.4 / 100,000$ women $[21,22]$. The presenting symptoms of this tumor type greatly overlap with those of benign ULs, making early diagnosis of ULMSs difficult. Surgery is the recommended primary treatment, yet the diagnosis is often made histologically after the surgery. Even then, the clinical course of ULMS is difficult to predict. Occasionally, diagnostic challenges emerge in daily pathological practice in distinguishing ULMSs from histopathological UL subtypes. The accurate diagnosis has important prognostic and therapeutic implications. Most ULMSs are aneuploid with complex structural chromosomal alterations [23], however, no consistent structural aberrations have been identified in these tumors. So far, only a few genes have been associated with ULMSs, including TP53, RB1, ATRX, and MED12 [23, 24].

The aim of this study was to examine the frequency of known genetic leiomyoma driver alterations in histopathological UL subtypes and ULMSs to scrutinize molecular variability in these tumors and to identify a potential UL subtype(s) that resembles ULMSs. The study material consisted of a comprehensive series of 94 histopathological UL variants (incl. leiomyomas with bizarre nuclei, cellular, and mitotically active leiomyomas), 51 ULMSs, and 65 conventional ULs as controls.
MED12 mutation status was analyzed by direct sequencing, while HMGA2 overexpression and biallelic $F H$ inactivation were determined by immunohistochemistry.

\section{Methods \\ Samples}

A retrospective series of 210 archival formalin-fixed paraffin-embedded (FFPE) samples representing various uterine smooth muscle tumors was collected at the Department of Pathology, Hospital District of Helsinki and Uusimaa, Helsinki, Finland, after which the samples were anonymized for the study. The series included 65 conventional, 51 cellular ( 22 cellular and 29 highly cellular), and 25 mitotically active leiomyomas (11 lesions showing simultaneously increased cellularity), 18 leiomyomas with bizarre nuclei, and 51 leiomyosarcomas.

\section{Histological evaluation}

Histological assessment of each specimen was performed by a pathologist specialized in gynecological pathology (RB) and the tumors were classified into conventional, cellular, highly cellular, and mitotically active leiomyomas, leiomyomas with bizarre nuclei, and ULMSs according to the WHO criteria [4] (see Additional file 1: Table S1). The microscopic morphology of highly cellular leiomyoma may mimic endometrial stromal tumor. In ambiguous cases, the differential diagnosis was aided by smooth muscle actin, desmin, h-caldesmon (smooth muscle markers), and CD10 (relative endometrial stromal cell marker) immunohistochemistry. For DNA extraction and tissue microarray (TMA) construction, pathologist marked representative tumor regions of each sample.

\section{DNA extraction and tissue microarray construction}

Genomic DNA was extracted either with NucleoSpin ${ }^{\circ}$ FFPE DNA Kit, NucleoSpin ${ }^{\circ}$ FFPE RNA/DNA Kit (MachereyNagel, Düren, Germany), or with a conventional nonenzymatic method [25]. Quadruplicate $0.8 \mathrm{~mm}$ cores of FFPE tumor tissue from each case were punched with a manual tissue arrayer (MTA-I, Beecher Instruments, Sun Prairie, WI, USA) to an empty paraffin block. Cores of FFPE myometrium tissue were also incorporated in the TMA block as internal controls.

\section{MED12 mutation analysis}

MED12 mutation screening of exons 1 and 2 was performed by direct sequencing. Exon 2, which harbors the great majority of mutations, was analyzed first and if negative, mutation screening was extended to exon 1. MED12 exon 2 mutation status has been previously reported for 64 out of 65 conventional ULs, 91 out of 94 histopathological UL variants, and 19 out of 51 ULMSs [12, 24, 26], and exon 1 for all MED12 exon 2 mutation-negative conventional ULs $(n=28)$ [26] and histopathological UL 
variants $(n=77)$ [27]. The primer sequences and conditions have been previously described [12, 27]. Sequencing was carried out using Big Dye Terminator v3.1 sequencing chemistry (Applied Biosystems, Foster City, CA, USA) on an ABI3730 automatic DNA Sequencer according to the manufacturer's instructions. The sequence graphs were analyzed both manually and with Mutation Surveyor software (SoftGenetics, State College, PA, USA). MED12 NM_005120.2 was used as a reference sequence. MED12 mutation screening was successful in $98.6 \%(207 / 210)$ of the samples for exon 2 and in 95.2\% (138/145) for exon 1. The three samples which failed in direct sequencing of exon 2 showed clearly visible larger deletions on an agarose gel (see Additional file 2: Figure S1).

\section{Immunohistochemistry}

HMGA2 overexpression and biallelic $F H$ inactivation were assessed by immunohistochemistry. Biallelic inactivation of $F H$ was detected with 2 -succinocysteine (2SC) staining, which is based on the recognition of modified (succinated) proteins formed in FH-deficient cells as a result of the accumulation of fumarate [28, 29]. 2SC status has been previously reported for all 65 conventional ULs [26]. Heat-induced antigen retrieval was carried out in a microwave using citrate buffer ( $\mathrm{pH}$ 6.0). Endogenous peroxidase blocking was followed by overnight incubation with the primary antibody at $4{ }^{\circ} \mathrm{C}$ (antiHMGA2 1:2000, Biocheck Inc., Foster City, CA, USA; anti-2SC 1:2000 [29]). A positive reaction for HMGA2 and 2SC was detected by DAB Plus Substrate System (Thermo Fisher Scientific, Waltham, MA, USA) or EnVision + kit (Dako, Carpinteria, CA, USA), respectively.

\section{Scoring of the TMAs}

Immunohistochemical scoring was carried out by a pathologist (RB). For both HMGA2 and 2SC, the intensity of the immunoreaction was classified into four groups: - = fully negative, $(+)=$ single cell positivity, $+=$ low positivity, $++=$ strong positivity (see Additional file 3: Figure S2). Only samples that showed strong positivity in the immunoreaction were considered positive. For 2SC, the positive staining indicated accumulation of fumarate and succinated proteins, and thus biallelic inactivation of $F H$, while the negative staining indicated that the cells retained sufficient amount of FH. For HMGA2, only nuclear labelling of the protein was evaluated.

\section{$\mathrm{FH}$ mutation analysis}

Tumors showing 2 SC positivity $(n=10)$ were directly sequenced for $F H$ mutations. The sequencing was carried out as described above in MED12 mutation analysis. $F H$ NM_000143.3 was used as a reference sequence. $F H$ exon 1 was excluded from the mutation screening. Two out of ten 2SC-positive tumors, both representing ULMSs, did not amplify at all and were thus excluded from the mutation screening.

\section{Statistical analyses}

Statistical analyses were performed using $\mathrm{R}$ software, version 2.14.0 ( $\mathrm{R}$ Foundation for Statistical Computing, Vienna, Austria, www.r-project.org). Fisher's exact test was used to compare the frequency of MED12 mutations, presence of $H M G A 2$ overexpression and biallelic $F H$ inactivation between conventional ULs and histopathological UL subtypes or ULMSs. Eleven mitotically active leiomyomas with increased cellularity were included in both mitotically active and cellular/highly cellular leiomyoma subtypes for statistical testing. All $P$-values were two-sided and $P$-value $<0.05$ was considered statistically significant.

\section{Findings}

Uterine smooth muscle tumor classification

Based on the number of mitotic figures per 10 high power fields, severity of nuclear atypia (0-3), degree of cellularity (normal, cellular, highly cellular), and presence of tumor necrosis in the hematoxylin-eosin-stained sections of the tumor specimens, the samples were classified into 65 conventional, 51 cellular (22 cellular and 29 highly cellular), and 25 mitotically active leiomyomas (11 lesions showing simultaneously increased cellularity), 18 leiomyomas with bizarre nuclei, and 51 leiomyosarcomas (see Additional file 4: Figure S3).

\section{Conventional leiomyomas}

MED12 and HMGA2 alterations accounted for the vast majority of conventional ULs $(53 / 65,81.5 \%)$ (Table 1, Fig. 1, see Additional file 5: Table S2), which is in line with previous literature [11]. The third driver alteration, biallelic $F H$ inactivation, is known to be very rare in sporadic conventional ULs [15, 26, 30, 31]. Accordingly, all conventional ULs included in this study were FH proficient [26]. This set of conventional ULs provides thus an appropriate reference series for the other tumor types in this study.

\section{Histopathological uterine leiomyoma subtypes}

As previously reported, the histopathological UL variants $(18.1 \%, 17 / 94)$ harbored significantly fewer MED12 mutations than the conventional leiomyomas (56.9\%, 37/65; $P=5.2 \times 10^{-7}$ ) [12] (Table 1, see Additional file 5: Table S2 and Additional file 6: Table S3). No significant difference was seen in the number of HMGA2 overexpressing tumors between histopathological UL variants (13.8\%, 13/ 94) and conventional leiomyomas (Table 1, see Additional file 5: Table S2 and Additional file 6: Table S3). Unlike conventional leiomyomas, the histopathological UL variants $(8.5 \%, 8 / 94)$ displayed 2SC positivity as a sign of $\mathrm{FH}$ deficiency (Table 1, Fig. 1, see Additional file 5: Table S2 and Additional file 6: Table S3). 
Table 1 MED12 mutations, HMGA2 aberrations, and biallelic FH inactivation in uterine smooth muscle tumors

\begin{tabular}{|c|c|c|c|c|c|c|c|c|c|c|c|c|c|}
\hline \multirow[t]{2}{*}{ Smooth muscle tumor subtype } & \multirow[t]{2}{*}{ Total } & \multicolumn{4}{|c|}{ MED12 mutation positive } & \multicolumn{4}{|c|}{ HMGA2 overexpressing } & \multicolumn{4}{|c|}{ FH deficient } \\
\hline & & $\mathrm{N}$ & $\%$ & $P$-value & $95 \% \mathrm{Cl}$ & $\mathrm{N}$ & $\%$ & $P$-value & $95 \% \mathrm{Cl}$ & $\mathrm{N}$ & $\%$ & $P$-value & $95 \% \mathrm{Cl}$ \\
\hline Conventional & 65 & 37 & 56.9 & & & 16 & 24.6 & & & 0 & 0 & & \\
\hline Histopathological UL variant & 94 & 17 & 18.1 & $5.2 \times 10^{-7}$ & $2.76-13.14$ & 13 & 13.8 & 0.10 & $0.83-5.01$ & 8 & 8.5 & 0.02 & $0.00-0.81$ \\
\hline Mitotically active & 25 & 9 & 36 & 0.10 & $0.83-6.93$ & 1 & 4 & 0.03 & $1.07-341.56$ & 0 & 0 & & \\
\hline Leiomyoma with bizarre nuclei & 18 & 3 & 16.7 & $3.0 \times 10^{-3}$ & $1.61-38.18$ & 0 & 0 & 0.02 & $1.24-\infty$ & 6 & 33.3 & $4.9 \times 10^{-5}$ & $0.00-0.19$ \\
\hline Cellular & 25 & 4 & 16 & $7.3 \times 10^{-4}$ & $1.98-30.31$ & 8 & 32 & 0.60 & $0.23-2.23$ & 1 & 4 & 0.28 & $0.00-15.00$ \\
\hline Highly cellular & 37 & 3 & 8.1 & $6.0 \times 10^{-7}$ & $3.98-81.73$ & 4 & 10.8 & 0.12 & $0.77-11.96$ & 1 & 2.7 & 0.36 & $0.00-22.2$ \\
\hline Leiomyosarcoma & 51 & 11 & 21.6 & $1.4 \times 10^{-4}$ & $1.96-12.16$ & 3 & 5.9 & 0.01 & $1.35-29.40$ & 2 & 3.9 & 0.19 & $0.00-4.16$ \\
\hline
\end{tabular}

Eleven mitotically active leiomyomas with increased cellularity were included in both mitotically active and cellular/highly cellular leiomyoma subtypes for statistical testing

\section{Mitotically active leiomyomas}

Mitotically active ULs occur primarily in premenopausal women. Tumors display an increased number of mitotic figures per 10 high power fields, but otherwise present macroscopic and histologic appearances of conventional leiomyomas, lacking cytological atypia and tumor cell
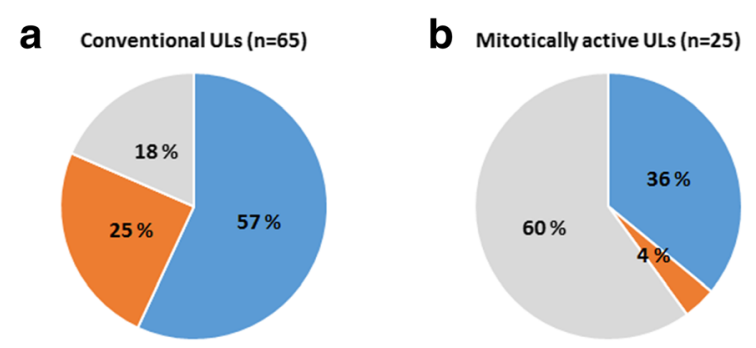

C

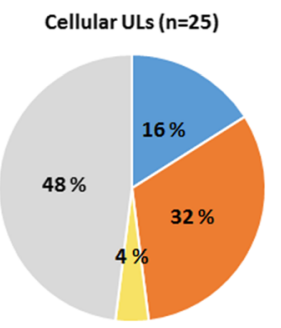

e
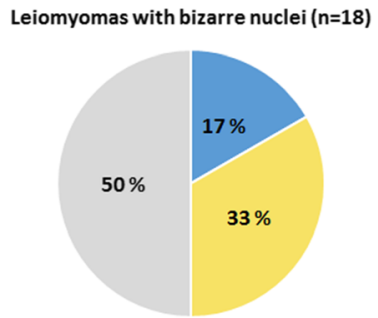

Fig. 1 Mutation spectra of uterine smooth muscle tumors. The frequencies of MED12 mutations, HMGA2 aberrations, and biallelic FH inactivation in conventional ULS $\mathbf{a}$, histopathological UL variants $\mathbf{b}-\mathbf{e}$, and ULMSs $\mathbf{f}$. Eleven mitotically active leiomyomas with increased cellularity were included in both mitotically active and cellular/highly cellular leiomyoma subtypes necrosis [32, 33]. Hormonal and other external factors in the tumor microenvironment have been associated with this tumor type and are thought to contribute to the elevated number of mitoses. For example, mitotically active ULs are usually diagnosed during the secretory phase of the menstrual cycle [34] and patients using progestin display significantly higher mitotic activity in their leiomyomas compared to control patients [35]. The known leiomyoma driver alterations were observed in $40 \%$ of the tumors: MED12 mutation frequency was slightly, but not significantly, lower in mitotically active ULs (36\%, 9/25) compared to conventional leiomyomas (Table 1, Fig. 1, see Additional file 5: Table S2 and Additional file 6: Table S3). Other alterations were very rare as only one tumor showed HMGA2 overexpression (4\%) and none displayed biallelic FH inactivation (Table 1, Fig. 1, see Additional file 6: Table S3). Our results suggest that the molecular background of mitotically active ULs slightly differs from that of conventional ULs.

\section{Cellular leiomyomas}

Cellular leiomyomas present clearly increased cellularity when compared to the surrounding myometrium, lack nuclear atypia and tumor necrosis, and have infrequent mitotic figures [36]. The tumors commonly have an irregular border that merges with the surrounding myometrium. Cytogenetically, these tumors have been shown to display loss of the short arm of chromosome 1, and their transcriptional profile has been suggested to be more similar to leiomyosarcomas than conventional leiomyomas or normal myometrium [37]. Additionally, two small clinical series have reported cellular leiomyomas to associate with aggressive clinical behavior [38, 39]. Based on our results, the most common driver alteration in cellular ULs is HMGA2 overexpression, which was seen in one third of the tumors $(32 \%, 8 / 25)$ (Table 1, Fig. 1, see Additional file 6: Table S3). This was the highest frequency of HMGA2 overexpression in any tumor type studied. The difference was statistically significant when 
compared to mitotically active ULs, leiomyomas with bizarre nuclei, or ULMSs. The MED12 mutation frequency in these tumors was rather low: $16 \%(4 / 25)$ (Table 1, Fig. 1, see Additional file 6: Table S3). Also one cellular UL that presented $2 \mathrm{SC}$ positivity (4\%) and harbored a potential $\mathrm{FH}$ mutation in exon 3 (c.321_323del3, p.N107_Q108delinsL) was identified. This three base pair deletion results in the substitution of amino acids N107 and Q108 with leucine (L). Missense mutation affecting the same amino acid (c.320A > C, p.N107T) has been reported in six UK HLRCC families [14], supporting the pathogenicity of the mutation. Overall, the leiomyoma driver alterations were observed in more than half of the cellular ULs $(52 \%, 13 /$ 25), but their distribution differed from that observed in conventional tumors.

\section{Highly cellular leiomyomas}

Highly cellular leiomyomas are characterized with even higher density of cells reminiscent of endometrial stromal tumors, and may be occasionally misdiagnosed as endometrial stromal nodules when well circumscribed $[36,40]$. Here, these tumors harbored the lowest number of MED12 mutations of all tumor types analyzed (8.1\%, 3/ 37) (Table 1, Fig. 1, see Additional file 6: Table S3). Four tumors overexpressed HMGA2 (10.8\%) and one showed 2SC positivity (2.7\%). Overall, this tumor subtype displayed the least amount of leiomyoma driver alterations $(21.6 \%, 8 / 37)$ suggesting that there are other, still unknown factors underlying the genesis of these lesions.

\section{Leiomyomas with bizarre nuclei}

Leiomyomas with bizarre nuclei (previously termed 'atypical leiomyomas') are characterized by the presence of large cells with eosinophilic cytoplasm and bizarrely shaped, multilobated or -nucleated nuclei. Low mitotic activity and lack of tumor cell necrosis are also typical for these tumors [41, 42]. Interestingly, as many as one third of ULs with bizarre nuclei $\left(33.3 \%, 6 / 18 ; P=4.9 \times 10^{-5}\right)$ displayed 2SC positivity, indicating biallelic $F H$ inactivation (Table 1, Fig. 1, see Additional file 6: Table S3). Subsequently, our sequencing efforts identified an $F H$ mutation in two of the tumors. One mutation, c.587A > G, p.H196R, has been previously reported in three Finnish HLRCC families [30, 43] and in three isolated cases. The germline origin of the mutation was confirmed also for the patient in this study. Altogether six patients in these previously identified families have been diagnosed with renal cell cancer. Another tumor with bizarre nuclei had a mutation, c.739-2A > C in exon-intron junction preceding exon 6, which likely affects splicing. FH deficiency has been shown to be overrepresented in leiomyomas with bizarre nuclei and some of the morphologic features typical for these tumors have been associated with $F H$ mutations [42, 44-47]. Even though morphologic features alone lack robustness to separate sporadic and HLRCC-associated ULs [48-50], the possibility of an underlying germline $F H$ mutation should be considered in patients with ULs with bizarre nuclei. Clinical features such as the severity of symptoms, number of tumors within the uterus, age at diagnosis, and possible family history of ULs can be evaluated, and patients should be examined for possible cutaneous leiomyomas. Should additional features suggestive of HLRCC be identified, individuals could be directed to genetic counselling and molecular screening. Subsequently, mutation-positive individuals could be informed on the possible negative effects of HLRCC on fertility, and offered appropriate screening program for renal cell cancer. On the other hand, FH deficiency can also be a somatic event in ULs and may not exclusively indicate HLRCC syndrome, although somatic biallelic $F H$ inactivation has only rarely been reported [31, 50]. The most prevalent alterations in conventional ULs were only rarely observed in leiomyomas with bizarre nuclei: MED12 mutations were identified in three out of 18 tumors (16.7\%) and none displayed HMGA2 overexpression (Table 1, Fig. 1, see Additional file 6: Table S3). Recently, it has been suggested that leiomyomas with bizarre nuclei can be divided into two subtypes based primarily on nuclear features; some tumors show significantly higher rates of HMGA2 immunoreactivity and frequent MED12 mutations and the others may occasionally be related to $F H$ mutations [51]. The high frequency of $F H$ inactivation and the low frequency of MED12 and HMGA2 alterations distinctly separates these tumors from conventional ULs, other histopathological UL variants, and ULMSs.

\section{Uterine leiomyosarcomas}

Leiomyosarcomas are diagnosed primarily based on cytological atypia, mitotic activity, and tumor cell necrosis, which distinguishes them from benign uterine smooth muscle tumors [52]. Despite well-defined morphological criteria, diagnostic challenges emerge, at times, in daily pathological practice in differentiating ULMSs from histopathological UL subtypes. Leiomyosarcomas are known to harbor complex numerical and structural chromosomal aberrations, as well as mutations in well-known cancer genes $A T R X, T P 53$, and $R B 1$ [23, 24, 53]. As previously reported, $M E D 12$ mutations were one of the most common alterations in ULMSs $(21.6 \%, 11 / 51)$, though significantly less frequent than in conventional ULs $\left(P=1.4 \times 10^{-4}\right)$ (Table 1, Fig. 1, see Additional file 5: Table S2 and Additional file 7: Table S4). [24, 26, 54]. Also HMGA2 overexpression was infrequent compared to conventional ULs $(5.9 \%, 3 / 51$, $P=0.01$ ) and two tumors showed biallelic $F H$ inactivation (3.9\%). Although not as common as in conventional ULs, leiomyoma driver alterations were observed in nearly one third of ULMSs $(31.4 \%, 16 / 51)$. This suggests that some ULMSs may originate from a leiomyoma precursor, or that 
the UL driver alterations provide a growth advantage also for these malignant smooth muscle tumors. The progression from a benign UL precursor to a malignant leiomyosarcoma has been proposed also previously based on microscopically visible co-localization of morphologically benign areas within ULMS [13, 55-57]. Furthermore, in some of these cases, identical MED12 mutations have been identified in both components [13]. Of note, one leiomyosarcoma FFPE block in our sample series showed simultaneous co-localization of a leiomyoma with bizarre nuclei. In this case, both tumors were wild type for all leiomyoma driver alterations.

\section{Mutual exclusivity}

Altogether 65 uterine smooth muscle tumors harbored MED12 mutations, 32 showed HMGA2 overexpression, and ten 2SC positivity, indicating biallelic $F H$ inactivation. None of the tumors displayed simultaneously more than one leiomyoma driver alteration, indicating that MED12 mutations, HMGA2 overexpression, and biallelic $F H$ inactivation are mutually exclusive (Fig. 2).

\section{Limitations of the study}

The differentiation of uterine smooth muscle tumors may occasionally result in ambiguous cases, which are challenging to diagnose. Although the sample series in this study

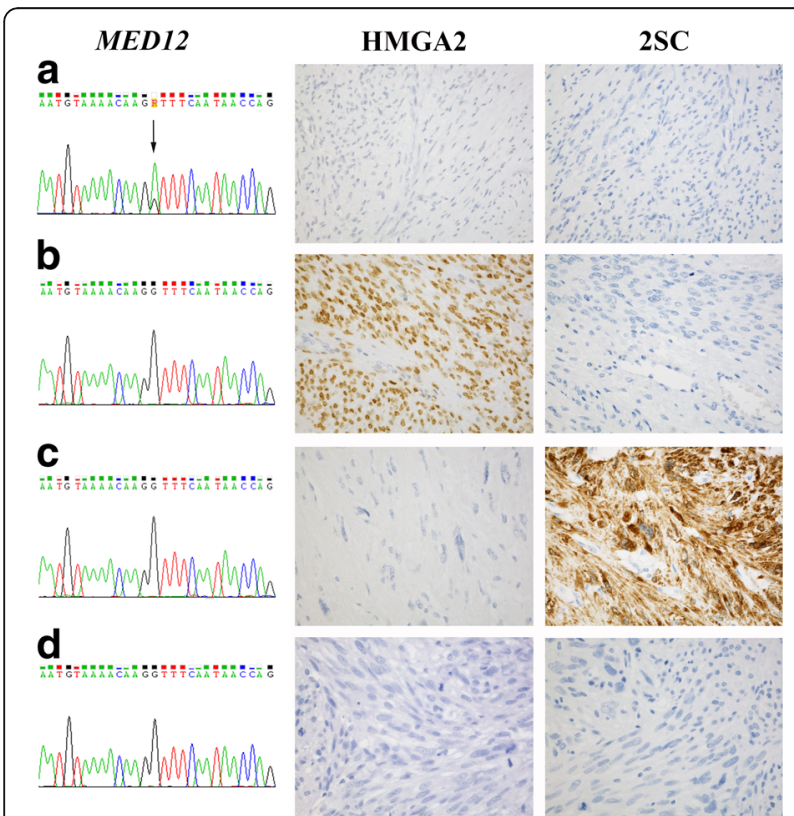

Fig. 2 Mutual exclusivity of MED12 mutations, HMGA2 overexpression, and biallelic FH inactivation. Representative figures of uterine smooth muscle tumors with different molecular genetic features. a MED12 mutation-positive conventional leiomyoma, b cellular leiomyoma with HMGA2 overexpression, c 2SC-positive leiomyoma with bizarre nuclei, and $\mathbf{d}$ leiomyosarcoma without the known genetic leiomyoma driver aberrations. Antibody stainings are shown with $\times 40$ magnification was large, considering the rarity of histopathological UL subtypes and ULMSs, the classification of tumors based on the leiomyoma driver alterations resulted in rather small subgroups. Also no clinical data were available for the samples. In the future, it would be interesting to study the associations between different tumor subgroups and clinical characteristics in an even larger set of samples. In addition, the fairly modest success rate of FH screening may be due to the low quantity and suboptimal quality of FFPE samples, or there might be other kinds of variations that contribute to the genesis of these lesions, such as larger deletions, insertions, or more complex structural alterations, which are not detectable by amplicon sequencing, or alternatively the alterations may be epigenetic or affect non-coding regulatory regions.

\section{Conclusions}

To conclude, 107 out of 210 uterine smooth muscle tumors analyzed harbored one of the three leiomyoma driver alterations. No tumor displayed more than one change, confirming the previous observations $[12,16$, $26,58]$ that these mutations are mutually exclusive driver alterations. While these alterations were identified in various UL subtypes, their relative frequencies varied considerably. Of particular interest was the high frequency of $\mathrm{FH}$-deficient tumors among leiomyomas with bizarre nuclei; although not an unambiguous indicator for HLRCC, the possibility of this highly penetrant tumor predisposition syndrome should be considered and additional suggestive clinical characteristics evaluated when these tumors are encountered at the clinic. Except for conventional ULs, a significant proportion of all other UL subtypes displayed no driver alterations and additional work is required to reveal the underlying causes in these mutationnegative tumors. Finally, nearly one third of ULMSs displayed one of the three UL driver alterations, emphasizing the role of these aberrations also in the ULMS tumorigenesis. It is clinically relevant to understand the molecular mechanisms driving the tumorigenesis of uterine smooth muscle tumors, as it may lead to improved diagnosis and personalized medical treatments in the future.

\section{Additional files}

Additional file 1: Table S1. Histopathological features of 210 uterine smooth muscle tumors (XLSX $19 \mathrm{~kb}$ ).

Additional file 2: Figure S1. Large deletions in MED12 exon 2. Three uterine smooth muscle tumors (two conventional leiomyomas and one leiomyosarcoma) harbor large MED12 exon 2 deletions (PDF 175 kb).

Additional file 3: Figure S2. Scoring of HMGA2 and 2SC antibody stainings. Representative figures of uterine smooth muscle tumors showing different intensities of the immunoreaction for HMGA2 and 
2SC $(-/(+) /+/++)$. Antibody stainings are shown with $\times 40$ magnification (PDF $216 \mathrm{~kb}$ )

Additional file 4: Figure S3. Representative hematoxylin-eosin stainings of uterine smooth muscle tumor subtypes. HE-stainings are shown with $\times 40$ magnification (PDF 364 kb).

Additional file 5: Table S2. MED12 exon 1/2 mutation status and results of HMGA2 and $2 \mathrm{SC}$ immunohistochemistry of 65 conventional uterine leiomyomas (XLSX $11 \mathrm{~kb})$.

Additional file 6: Table S3 MED12 exon 1/2 mutation status and results of HMGA2 and 2SC immunohistochemistry of 94 histopathological uterine leiomyoma variants (XLSX $13 \mathrm{~kb}$ ).

Additional file 7: Table S4. MED12 exon 1/2 mutation status and results of HMGA2 and 2SC immunohistochemistry of 51 uterine leiomyosarcomas (XLSX $12 \mathrm{~kb}$ ).

\section{Abbreviations}

2SC: 2-succinocysteine; ATRX: Alpha thalassemia/mental retardation syndrome X-linked; FFPE: Formalin-fixed paraffin-embedded; FH: Fumarate hydratase; HLRCC: Hereditary leiomyomatosis and renal cell cancer; HMGA2: High mobility group AT-hook 2; MED12: Mediator complex subunit 12; RB1: Retinoblastoma 1; TMA: Tissue microarray; TP53: Tumor protein P53; UL: Uterine leiomyoma; ULMS: Uterine leiomyosarcoma

\section{Acknowledgements}

We express our gratitude to Sini Nieminen for the help with the samples, and Gynel Arifdshan, lina Vuoristo, Inga-Lill Svedberg, and Alison Ollikainen for technical assistance.

\section{Funding}

This study was supported by the Academy of Finland (NM: 295693, PV: 260370 and 292769), the Sigrid Jusélius Foundation, and the Cancer Society of Finland.

\section{Availability of data and materials}

The datasets supporting the conclusions of this article are included within the article and its additional files.

\section{Authors' contributions}

NM and PV designed and conceived the study. NM and KK carried out the experiments. NM and RB participated in acquisition, analysis, and interpretation of data. NF participated in providing and analyzing the data. NM and PV wrote the manuscript. All authors participated in critical revision of the manuscript and in final approval of the submitted version.

\section{Competing interests}

The authors declare that they have no competing interests.

\section{Consent for publication}

Not applicable.

\section{Ethics approval and consent to participate}

All samples in this study were derived after authorization from the National Supervisory Authority for Welfare and Health (8522/06.01.03.01/2015). The study was approved by the appropriate ethics review board of the Hospital District of Helsinki and Uusimaa (HUS), Helsinki, Finland (88/13/03/03/2015).

\section{Publisher's Note}

Springer Nature remains neutral with regard to jurisdictional claims in published maps and institutional affiliations.

\section{Author details}

${ }^{1}$ Research Programs Unit, Genome-Scale Biology Research Program and Medicum, Department of Medical and Clinical Genetics, FIN-00014 University of Helsinki, P.O. Box 63, Helsinki, Finland. ²Department of Pharmacology, Physiology and Neuroscience, USC School of Medicine, University of South Carolina, Columbia, SC 29209, USA. ${ }^{3}$ Department of Pathology, Laboratory of Helsinki University Hospital (HUSLAB), Helsinki University Hospital and Medicum, FIN-00014 University of Helsinki, P.O. Box 21, Helsinki, Finland.
Received: 29 August 2016 Accepted: 1 June 2017

Published online: 07 June 2017

\section{References}

1. Stewart EA. Clinical practice. Uterine fibroids. N Engl J Med. 2015;372:1646-55.

2. Baird DD, Dunson DB, Hill MC, Cousins D, Schectman JM. High cumulative incidence of uterine leiomyoma in black and white women: ultrasound evidence. Am J Obstet Gynecol. 2003;188:100-7.

3. Cardozo ER, Clark AD, Banks NK, Henne MB, Stegmann BJ, Segars JH. The estimated annual cost of uterine leiomyomata in the United States. Am J Obstet Gynecol. 2012;206:211.e1-9.

4. Oliva E, Carcangiu ML, Carinelli SG, Ip P, Loening T, Longacre TA, et al. Mesenchymal tumours. In: Kurman RJ, Carcangiu ML, Herrington CS, Young $\mathrm{RH}$, editors. WHO classification of Tumours of female reproductive organs. 4th ed. Lyon: IARC Press; 2014. p. 135-47.

5. Nibert M, Heim S. Uterine leiomyoma cytogenetics. Genes Chromosomes Cancer. 1990:2:3-13.

6. Rein MS, Friedman AJ, Barbieri RL, Pavelka K, Fletcher JA, Morton CC. Cytogenetic abnormalities in uterine leiomyomata. Obstet Gynecol. 1991;77:923-6.

7. Meloni AM, Surti U, Contento AM, Davare J, Sandberg AA. Uterine leiomyomas: cytogenetic and histologic profile. Obstet Gynecol. 1992; 80:209-17.

8. Mäkinen N, Mehine M, Tolvanen J, Kaasinen E, Li Y, Lehtonen HJ, et al. MED12, the mediator complex subunit 12 gene, is mutated at high frequency in uterine leiomyomas. Science. 2011:334:252-5.

9. Je EM, Kim MR, Min KO, Yoo NJ, Lee SH. Mutational analysis of MED12 exon 2 in uterine leiomyoma and other common tumors. Int J Cancer. 2012;131:E1044-7.

10. McGuire MM, Yatsenko A, Hoffner L, Jones M, Surti U, Rajkovic A. Whole exome sequencing in a random sample of north American women with leiomyomas identifies MED12 mutations in majority of uterine leiomyomas. PLoS One. 2012;7:e33251.

11. Mehine M, Mäkinen N, Heinonen HR, Aaltonen LA, Vahteristo P. Genomics of uterine leiomyomas: insights from high-throughput sequencing. Fertil Steril. 2014;102:621-9.

12. Mäkinen $N$, Vahteristo $P$, Kämpjärvi K, Arola J, Butzow R, Aaltonen LA. MED12 exon 2 mutations in histopathological uterine leiomyoma variants. Eur J Hum Genet. 2013:21:1300-3.

13. Matsubara A, Sekine S, Yoshida M, Yoshida A, Taniguchi H, Kushima R, et al. Prevalence of MED12 mutations in uterine and extrauterine smooth muscle tumours. Histopathology. 2013;62:657-61.

14. Tomlinson IP, Alam NA, Rowan AJ, Barclay E, Jaeger EE, Kelsell D, et al. Germline mutations in $\mathrm{FH}$ predispose to dominantly inherited uterine fibroids, skin leiomyomata and papillary renal cell cancer. Nat Genet. 2002;30:406-10.

15. Mehine M, Kaasinen E, Mäkinen N, Katainen R, Kämpjärvi K, Pitkänen E, et al. Characterization of uterine leiomyomas by whole-genome sequencing. $\mathrm{N}$ Engl J Med. 2013;369:43-53.

16. Mehine M, Kaasinen E, Heinonen HR, Mäkinen N, Kämpjärvi K, Sarvilinna N, et al. Integrated data analysis reveals uterine leiomyoma subtypes with distinct driver pathways and biomarkers. Proc Natl Acad Sci U S A. 2016;113:1315-20.

17. Major FJ, Blessing JA, Silverberg SG, Morrow CP, Creasman WT, Currie JL, et al. Prognostic factors in early-stage uterine sarcoma. A gynecologic oncology group study. Cancer. 1993;71:1702-9.

18. Mayerhofer K, Obermair A, Windbichler G, Petru E, Kaider A, Hefler L, et al. Leiomyosarcoma of the uterus: a clinicopathologic multicenter study of 71 cases. Gynecol Oncol. 1999;74:196-201.

19. Dinh TA, Oliva EA, Fuller AF Jr, Lee H, Goodman A. The treatment of uterine leiomyosarcoma. Results from a 10-year experience (1990-1999) at the Massachusetts General Hospital. Gynecol Oncol. 2004:92:648-52.

20. Abeler VM, Royne O, Thoresen S, Danielsen HE, Nesland JM, Kristensen GB. Uterine sarcomas in Norway. A histopathological and prognostic survey of a total population from 1970 to 2000 including 419 patients. Histopathology. 2009;54:355-64.

21. Toro JR, Travis LB, Wu HJ, Zhu K, Fletcher CD, Devesa SS. Incidence patterns of soft tissue sarcomas, regardless of primary site, in the surveillance, epidemiology and end results program, 1978-2001: an analysis of 26,758 cases. Int J Cancer. 2006;119:2922-30.

22. Koivisto-Korander R, Martinsen Jl, Weiderpass E, Leminen A, Pukkala E. Incidence of uterine leiomyosarcoma and endometrial stromal sarcoma in 
Nordic countries: results from NORDCAN and NOCCA databases. Maturitas. 2012;72:56-60.

23. Sandberg AA. Updates on the cytogenetics and molecular genetics of bone and soft tissue tumors: leiomyosarcoma. Cancer Genet Cytogenet. 2005;161:1-19.

24. Mäkinen N, Aavikko M, Heikkinen T, Taipale M, Taipale J, Koivisto-Korander R, et al. Exome sequencing of uterine Leiomyosarcomas identifies frequent mutations in TP53, ATRX, and MED12. PLoS Genet. 2016;12:e1005850.

25. Lahiri DK, Nurnberger JI Jr. A rapid non-enzymatic method for the preparation of HMW DNA from blood for RFLP studies. Nucleic Acids Res. 1991;19:5444

26. Kämpjärvi K, Mäkinen N, Mehine M, Välipakka S, Uimari O, Pitkänen E, et al. MED12 mutations and FH inactivation are mutually exclusive in uterine leiomyomas. Br J Cancer. 2016;114:1405-11.

27. Kämpjärvi K, Park MJ, Mehine M, Kim NH, Clark AD, Bützow R, et al. Mutations in Exon 1 highlight the role of MED12 in uterine Leiomyomas. Hum Mutat. 2014:35:1136-41.

28. Nagai R, Brock JW, Blatnik M, Baatz JE, Bethard J, Walla MD, et al. Succination of protein thiols during adipocyte maturation: a biomarker of mitochondrial stress. J Biol Chem. 2007;282:34219-28.

29. Bardella C, El-Bahrawy M, Frizzell N, Adam J, Ternette N, Hatipoglu E, et al. Aberrant succination of proteins in fumarate hydratase-deficient mice and HLRCC patients is a robust biomarker of mutation status. J Pathol. 2011;225:4-11.

30. Kiuru M, Lehtonen R, Arola J, Salovaara R, Jarvinen H, Aittomaki K, et al. Few $\mathrm{FH}$ mutations in sporadic counterparts of tumor types observed in hereditary leiomyomatosis and renal cell cancer families. Cancer Res. 2002; 62:4554-7.

31. Lehtonen R, Kiuru M, Vanharanta S, Sjöberg J, Aaltonen LM, Aittomäki K, et al. Biallelic inactivation of fumarate hydratase (FH) occurs in nonsyndromic uterine leiomyomas but is rare in other tumors. Am J Pathol. 2004;164:1722.

32. O'Connor DM, Norris HJ. Mitotically active leiomyomas of the uterus. Hum Pathol. 1990;21:223-7.

33. Prayson RA, Hart WR. Mitotically active leiomyomas of the uterus. Am J Clin Pathol. 1992:97:14-20.

34. Kawaguchi K, Fujii S, Konishi I, Nanbu Y, Nonogaki H, Mori T. Mitotic activity in uterine leiomyomas during the menstrual cycle. Am J Obstet Gynecol. 1989;160:637-41.

35. Tiltman AJ. The effect of progestins on the mitotic activity of uterine fibromyomas. Int J Gynecol Pathol. 1985;4:89-96.

36. Oliva E, Young RH, Clement PB, Bhan AK, Scully RE. Cellular benign mesenchymal tumors of the uterus. A comparative morphologic and immunohistochemical analysis of 33 highly cellular leiomyomas and six endometrial stromal nodules, two frequently confused tumors. Am J Surg Pathol. 1995;19:757-68

37. Christacos NC, Quade BJ, Dal Cin P, Morton CC. Uterine leiomyomata with deletions of Ip represent a distinct cytogenetic subgroup associated with unusual histologic features. Genes Chromosomes Cancer. 2006:45:304-12.

38. Giuntoli RL 2nd, Gostout BS, CS DM, Metzinger DS, Keeney GL. Diagnostic criteria for uterine smooth muscle tumors: leiomyoma variants associated with malignant behavior. J Reprod Med. 2007;52:1001-10.

39. Taran FA, Weaver AL, Gostout BS, Stewart EA. Understanding cellular leiomyomas: a case-control study. Am J Obstet Gynecol. 2010;203:109.e1-6.

40. Pujani M, Jairajpuri ZS, Rana S, Jetley S, Hassan MJ, Jain R. Cellular leiomyoma versus endometrial stromal tumor: a pathologists' dilemma. J Midlife Health. 2015:6:31-4.

41. Downes KA, Hart WR. Bizarre leiomyomas of the uterus: a comprehensive pathologic study of 24 cases with long-term follow-up. Am J Surg Pathol. 1997;21:1261-70.

42. Ly A, Mills AM, McKenney JK, Balzer BL, Kempson RL, Hendrickson MR, et al. Atypical leiomyomas of the uterus: a clinicopathologic study of 51 cases. Am J Surg Pathol. 2013;37:643-9.

43. Lehtonen HJ, Kiuru M, Ylisaukko-Oja SK, Salovaara R, Herva R, Koivisto PA, et al. Increased risk of cancer in patients with fumarate hydratase germline mutation. J Med Genet. 2006:43:523-6.

44. Siti-Aishah MA, Noriah O, Malini MN, Zainul-Rashid MR, Das S. Atypical (symplastic) leiomyoma of the uterus-a case report. Clin Ter. 2011;162: 447-50.

45. Croce S, Young RH, Oliva E. Uterine leiomyomas with bizarre nuclei: a clinicopathologic study of 59 cases. Am J Surg Pathol. 2014;38:1330-9.
46. Reyes C, Karamurzin Y, Frizzell N, Garg K, Nonaka D, Chen YB, et al. Uterine smooth muscle tumors with features suggesting fumarate hydratase aberration: detailed morphologic analysis and correlation with S-(2-succino)cysteine immunohistochemistry. Mod Pathol. 2014;27:1020-7.

47. Miettinen M, Felisiak-Golabek A, Wasag B, Chmara M, Wang Z, Butzow R, et al. Fumarase-deficient uterine Leiomyomas: an Immunohistochemical, molecular genetic, and Clinicopathologic study of 86 cases. Am J Surg Pathol. 2016;40:1661-9.

48. Alsolami S, El-Bahrawy M, Kalloger SE, AIDaoud N, Pathak TB, Chung CT, et al. Current morphologic criteria perform poorly in identifying hereditary leiomyomatosis and renal cell carcinoma syndrome-associated uterine leiomyomas. Int J Gynecol Pathol. 2014;33:560-7.

49. Martinek P, Grossmann P, Hes O, Bouda J, Eret V, Frizzell N, et al. Genetic testing of leiomyoma tissue in women younger than 30 years old might provide an effective screening approach for the hereditary leiomyomatosis and renal cell cancer syndrome (HLRCC). Virchows Arch. 2015;467:185-91.

50. Harrison WJ, Andrici J, Maclean F, Madadi-Ghahan R, Farzin M, Sioson L, et al. Fumarate Hydratase-deficient uterine Leiomyomas occur in both the Syndromic and sporadic settings. Am J Surg Pathol. 2016;40:599-607.

51. Ubago JM, Zhang Q, Kim JJ, Kong B, Wei JJ. Two subtypes of atypical Leiomyoma: clinical, Histologic, and molecular analysis. Am J Surg Pathol. 2016:40:923-33.

52. Toledo G, Oliva E. Smooth muscle tumors of the uterus: a practical approach. Arch Pathol Lab Med. 2008;132:595-605.

53. Fletcher JA, Morton CC, Pavelka K, Lage JM. Chromosome aberrations in uterine smooth muscle tumors: potential diagnostic relevance of cytogenetic instability. Cancer Res. 1990;50:4092-7.

54. Perot G, Croce S, Ribeiro A, Lagarde P, Velasco V, Neuville A, et al. MED12 alterations in both human benign and malignant uterine soft tissue tumors. PLoS One. 2012;7:e40015.

55. Zhang P, Zhang C, Hao J, Sung CJ, Quddus MR, Steinhoff MM, et al. Use of $X$-chromosome inactivation pattern to determine the clonal origins of uterine leiomyoma nd leiomyosarcoma. Hum Pathol. 2006;37:1350-6.

56. Mittal K, Joutovsky A. Areas with benign morphologic and immunohistochemical features are associated with some uterine leiomyosarcomas. Gynecol Oncol. 2007;104:362-5.

57. Mittal KR, Chen F, Wei JJ, Rijhvani K, Kurvathi R, Streck D, et al. Molecular and immunohistochemical evidence for the origin of uterine leiomyosarcomas from associated leiomyoma and symplastic leiomyomalike areas. Mod Pathol. 2009;22:1303-11.

58. Markowski DN, Bartnitzke S, Loning T, Drieschner N, Helmke BM, Bullerdiek J. MED12 mutations in uterine fibroids-their relationship to cytogenetic subgroups. Int J Cancer. 2012;131:1528-36.

\section{Submit your next manuscript to BioMed Central and we will help you at every step:}

- We accept pre-submission inquiries

- Our selector tool helps you to find the most relevant journal

- We provide round the clock customer support

- Convenient online submission

- Thorough peer review

- Inclusion in PubMed and all major indexing services

- Maximum visibility for your research

Submit your manuscript at www.biomedcentral.com/submit 